\title{
Correlation of Brownian Motions and Its Impact on a Reinsurer's Optimal Investment Strategy and Reinsured Proportion under Exponential Utility Maximization and Constant Elasticity of Variance Model
}

\author{
Silas A. Ihedioha \\ Department of Mathematics, Plateau State University Bokkos, Jos, Nigeria \\ Email: silasihedioha@yahoo.com
}

How to cite this paper: Ihedioha, S.A. (2018) Correlation of Brownian Motions and Its Impact on a Reinsurer's Optimal Investment Strategy and Reinsured Proportion under Exponential Utility Maximization and Constant Elasticity of Variance Model. Open Access Library Journal, 5: e4954.

https://doi.org/10.4236/oalib.1104954

Received: October 1, 2018

Accepted: October 27, 2018

Published: October 30, 2018

Copyright $\odot 2018$ by author and Open Access Library Inc.

This work is licensed under the Creative Commons Attribution International License (CC BY 4.0).

http://creativecommons.org/licenses/by/4.0/

\begin{abstract}
This work investigated a reinsurer's optimal investment strategy and the proportion he accepted for reinsurance under proportional reinsurance and exponential utility preference in the cases where the Brownian motions were correlated and where they did not correlate. The reinsurer invested in a market in which the price process of the risky asset is governed by constant elasticity of variance (CEV) model. The required Hamilton-Jacobi-Bellman Equations (HJB) were derived using the Ito's lemma from which the optimal investment strategy and reinsured proportion were calculated. Also investigated were the implications of the correlation coefficient.
\end{abstract}

\section{Subject Areas}

Financial Mathematics, Ordinary Differential Equation, Partial Differential Equation

\section{Keywords}

Correlation of Brownian Motions, Investment Strategy, Reinsured Proportion, Exponential Utility Constant Elasticity of Variance, Hamilton-Jacobi-Bellman Equation

\section{Introduction}

Among the social sciences which are designed for risk taking is insurance. On daily basis, people are exposed to infinitely many numbers of risks that affect 
their persons or their properties. The insured transfers his risks to an insurer, by paying a fixed cost called insurance premium to the reinsurer Also, insurers take insurance from another insurer, in this case called the reinsurer. Insurers and reinsurers use investment and reinsurance to balance their profit and risk. Reinsurance is a transaction where the reinsurer agrees to indemnify an insurer against all or part of the loss that the latter may sustain under a policy or policies that he has issued having been paid the required premium.

In literatures many contributors have studied the maximization of the utility of terminal value or minimizing the probability of ruin for the insurer of which Brown [1] made the contribution of giving an explicit solution to the problem of a firm that maximized the exponential utility of terminal wealth and minimized the probability of ruin with its surplus process given by Lundberg risk model.

Zhibin and Bayraktar [2] studied the problem of optimal reinsurance-investment problem in a constant elasticity of variance stock market for Jump-diffusion risk model obtaining the explicit expressions for the optimal strategies and value function whit they demonstrated using numerical examples to show the impact of model parameters on the optimal strategies.

Yang and Jiaqin [3] investigated the optimal investment-consumption-insurance with random parameter and discussed an optimal investment, consumption, and life insurance purchase problem for a wage earner in a complete market with a stream of Brownian motion.

Deng et al. [4] studied the optimal proportional reinsurance and investment for a constant elasticity of variance model under variance principle. They assumed that the insurer's surplus process followed a jump-diffusion process, the insurer can purchase proportional reinsurance from the reinsurer via the variance principle and invest in a risk-free asset and a risky asset whose price is modeled by a CEV model and obtain the techniques of stochastic control theory and closed-form expression for the value functions and optimal strategies.

Jianwei [5] considered the optimal investment strategy for annuity contracts under the constant elasticity of variance (CEV) model and derived the explicit solution for the power and exponential utility functions in two different periods, before and after retirement.

Ihedioha and Osu [6] worked on the optimal portfolios of an insurer and a reinsurer under proportional reinsurance and power utility preference in which the insurer's and the reinsurer's surplus processes were approximated by geometric Brownian motion with drift.

Li et al. [7] studied a time-consistent reinsurance-investment strategy for a mean-variance insurer under stochastic interest rate model and inflation risk and derived the time-consistent reinsurance-investment strategies as well as the corresponding value function for the mean-variance problem explicitly using numerical example.

Zhibin and Guo [8] considered the optimal proportional Reinsurance under two criteria-maximizing the expected utility and minimizing the value at risk and proved the existence and uniqueness of the optimal strategies and Pareto 
optimal solution, and gave the relationship between the optimal strategies.

$\mathrm{Gu}$ et al. [9] studied the constant elasticity of variance model for proportional reinsurance and investment strategies in which the claim process is assumed to follow a Brownian motion with drift, while the price process of the risky asset is described by the constant elasticity of variance model and obtain the optimal reinsurance and investment strategies.

In this study, we shall consider the case of a reinsurer assessing the impact of the correlation of the Brownian motions on the reinsurer's optimal investment strategy and reinsured proportion in the case the reinsurer's surplus process is approximated by constant elasticity of variance (CEV) model, and an insurer could purchase proportional reinsurance from the reinsurer.

We shall use the Ito's lemma to obtain the Hamilton-Jacob-Bellman (HJB) equation from which we shall obtain the reinsurer's optimal investment in the risky and the optimal reinsured proportion and investigate the correlation of the Brownian motions on them.

\section{The Model Formulation the Model}

The constant elasticity of variance (CEV) model is one-dimensional diffusion process that solves a stochastic differential equation (SDE). It is a natural extension of the geometric Brownian motion (GBM). The constant elasticity of variance model was originally proposed by Cox and Ross as an alternative diffusion process for European option pricing. Compared to Geometric Brownian Motion (GBM), we see that the advantages of the constant elasticity of variance (CEV) model are that the volatility rate has correlation with risky asset price and can explain the empirical bias such as volatility smile.

The constant elasticity of variance (CEV) model is giving as;

$$
\mathrm{d} S(t)=S(t)\left[\mu \mathrm{d} t+\beta S(t)^{\gamma} Z^{(2)}(t)\right]
$$

where

$\mu$ is a long term rate of return, $\gamma$ is the elasticity parameter satisfying $\gamma>0, \beta S(t)^{\gamma}$ is the volatility, and $Z^{(2)}(t)$ is a standard Brownian motion.

Remark: Note that when the elasticity parameter $\gamma$ equals zero, the constant elasticity of variance (CEV) model reduces to Geometric Brownian motion.

\section{The Model}

Let the claim process $C(t)$ of an insurance company be governed by

$$
\mathrm{d} C(t)=a \mathrm{~d} t-b \mathrm{~d} Z^{(1)}
$$

where $a$ and $b$ are positive constant and $Z^{(1)}(t)$ is a standard Brownian motion that is defined on a complete probability space $\left(\Omega, f,\left(f_{t}\right) ; t>0\right)$.

Let the premium rate be

$$
c=(1+\theta) a
$$

where $\theta>0$ is the security risk premium of an insurer who has obtained a 
reinsurance, then applying (3) in (2), the surplus process of the insurer is now given as

$$
\mathrm{d} R(t)=c \mathrm{~d} t-\mathrm{d} C(t)=a \theta \mathrm{d} t+b \mathrm{~d} Z^{(1)}(t)
$$

Assuming an insurer has the permission to purchase proportional reinsurance from a reinsure and pays premium on continuous basis, at the rate of $(1+\eta)$ ap $(t)$ where $\eta>\theta>0$ is the safety loading, then the surplus of the reinsurer is given as

$$
\mathrm{d} R(t)=\eta p(t) a \mathrm{~d} t+b p(t) \mathrm{d} Z^{(1)}(t)
$$

We also assume that both the insurer and the reinsurer invest their surpluses in the same market consisting of two assets; a risky asset (stock) and a riskless asset (bond). Let the prices of the risky and riskless asset be $S(t)$ and $B(t)$ respectively, and the price mechanism of the risky asset is driven by the constant elasticity of variance model, given a

$$
\mathrm{d} S(t)=S(t)\left[\mu d t+\beta S^{\gamma}(t) \mathrm{d} Z^{(2)}(t)\right], \gamma>0,0 \leq \beta \leq 1 .
$$

where $\mu$ denotes the appreciation rate of the risky asset and the $\beta S^{\gamma}(t)$ its volatility. $Z^{(2)}(t)$ is a standard Brownian motion defined on a complete probability space. The dynamics of the price of the riskless asset is given by the equation

$$
\mathrm{d} B(t)=k B(t) ; B(0)=1, k \text { a constant }
$$

Let $W(t)$ be the total money amount the reinsurer has for investment. If he invests $\pi(t)$ on the risky asset, then his investment on the riskless assets is $[W(t)-\pi(t)]$.

Corresponding to the policy $\pi$, the admissible strategy $[p(t), \pi(t)]$ the wealth process of the reinsurer evolve according to the stochastic differential Equations (SDEs);

$$
\mathrm{d} W^{\pi}(t)=\pi(t) \frac{\mathrm{d} S(t)}{S(t)}+[W(t)-\pi(t)] \frac{\mathrm{d} B(t)}{B(t)}+\mathrm{d} R(t)
$$

Substituting the expression for $\frac{\mathrm{d} S(t)}{S(t)}, \frac{\mathrm{d} B(t)}{B(t)}$, and $\mathrm{d} R(t)$, making use of Equations (1), (5), (6), and (7) respectively in Equations (8), we obtain,

$$
\begin{aligned}
\mathrm{d} W^{\pi}(t)= & \pi(t)\left[\mu \mathrm{d} t+\beta S^{\gamma}(t) \mathrm{d} Z^{(2)}(t)\right]+[W(t)-\pi(t)] k \mathrm{~d} t \\
& +\eta p(t) a \mathrm{~d} t+b p(t) \mathrm{d} Z^{(1)}
\end{aligned}
$$

Employing the rule

$$
\left.\begin{array}{l}
(\mathrm{d} t)^{2}=(\mathrm{d} t)\left(\mathrm{d} Z^{(1)}(t)\right)=(\mathrm{d} t)\left(\mathrm{d} Z^{(2)}(t)\right)=0, \\
{\left[\mathrm{~d} Z^{(1)}(t)\right]^{2}=\left[\mathrm{d} Z^{(2)}(t)\right]^{2}=\mathrm{d} t,} \\
{\left[\mathrm{~d} Z^{(1)}(t)\right]\left[\mathrm{d} Z^{(2)}(t)\right]=\rho \mathrm{d} t}
\end{array}\right\}
$$

the squared variation of the wealth process of the reinsurer is 


$$
\left(\mathrm{d} W^{\pi}\right)^{2}(t)=\left[\pi_{R}^{2}(t) \beta^{2} S^{2 \gamma}(t)+b^{2} p^{2}(t)\right] \mathrm{d} t,
$$

when the Brownian motions do not correlate and

$$
\left(\mathrm{d} W^{\pi}\right)^{2}(t)=\left[\pi^{2}(t)(t) \beta^{2} S^{2 \gamma}(t)+2 \beta b \rho S^{2 \gamma}(t) \pi(t)+b^{2} p^{2}(t)\right] \mathrm{d} t,
$$

when the Brownian motions correlate.

Suppose the reinsurer has exponential utility preferences given as

$$
U(w)=\frac{-\mathrm{e}^{-\phi w}}{\phi}, \phi>0,
$$

then the reinsurer's problem can then be written as

$$
V(T, w)=\operatorname{Max}_{\pi} E\left[U\left(W^{\pi}\right)\right] .
$$

subject to

$$
\begin{aligned}
\mathrm{d} W^{\pi}(t)= & \pi(t)\left[\mu \mathrm{d} t+\beta S^{\gamma}(t) \mathrm{d} Z^{(2)}(t)\right]+[W(t)-\pi(t)] k \mathrm{~d} t \\
& +\eta p(t) a \mathrm{~d} t+b p(t) \mathrm{d} Z^{(1)}
\end{aligned}
$$

\section{The Optimization Programme}

The optimal investment strategy and the reinsured are obtained in this section.

\subsection{The Case of None Correlation of Brownian Motions}

The application of Ito's lemma that states

$$
\mathrm{d} V=\frac{\partial V}{\partial w} \mathrm{~d} t+\frac{\partial V}{\partial w} \mathrm{~d} w+\frac{1}{2} \frac{\partial^{2}}{\partial w^{2}}(\mathrm{~d} w)^{2}
$$

we obtain the Hamilton-Jacobi-Bellman (HJB) equation

$V_{t}+\{(\mu-k) \pi(t)+k W(t)+a \eta p(t)\} V_{w}+\frac{\left[\pi^{2}(t) \beta^{2} S^{2 \gamma}(t)+b^{2} p^{2}(t)\right]}{2} V_{w w}=0$

where the rule

$$
E\left(\mathrm{~d} Z^{(1)}(t)\right)=E\left(\mathrm{~d} Z^{(2)}(t)\right)=0
$$

is applied.

In view of the homogeneity of the objective function, the restriction and the terminal condition, we make conjecture that the value function $V$ must be linear to $\frac{-\mathrm{e}^{-\phi w}}{\phi}$, so we let

$$
V(w, t, T)=h(t, T)\left(\frac{-\mathrm{e}^{-\phi w}}{\phi}\right)
$$

be such a function such that at the terminal date $T$,

$$
h(T, T)=1
$$

From (18), we obtain

$$
V_{t}=\frac{-\mathrm{e}^{-\phi w}}{\phi} h_{t}, V_{w}=\mathrm{e}^{-\phi w} h, V_{w w}=-\phi \mathrm{e}^{-\phi w}
$$


Using (20) in (16), we have

$$
\begin{aligned}
& \frac{-1}{\phi} h^{\prime}+\{(\mu-k) \pi(t)+k W(t)+a \eta p(t) \\
& \left.-\frac{\phi\left[\pi^{2}(t) \beta^{2} S^{2 \gamma}(t)(t)+b^{2} p^{2}(t)\right]}{2}\right\} h=0
\end{aligned}
$$

To obtain the optimal value $\pi^{*}(t)$ of $\pi(t)$ we differentiate (21) with respect to $\pi(t)$ to obtain

$$
(\mu-k)-\phi\left[\pi(t) \beta^{2} S^{2 \gamma}(t)\right]=0
$$

which simplifies to

$$
\pi^{*}(t)=\frac{(\mu-k)}{\phi \beta^{2} S^{2 \gamma}(t)}
$$

This is the reinsurer's optimal investment in the risky asset (stock) that is horizon dependent.

Also differentiating Equation (21) with respect to $p(t)$, we obtain

$$
a \eta-\phi b^{2} p(t)=0
$$

from which

$$
p^{*}(t)=\frac{a \eta}{\phi b^{2}}
$$

This is the optimal reinsured proportion. It is also dependent on time.

\subsection{The Case of Correlation of Brownian Motions}

We substitute (9) and (12) in (15) to obtain

$$
\begin{aligned}
\mathrm{d} V= & \frac{\partial V}{\partial t} \mathrm{~d} t+\frac{\partial V}{\partial w}\left\{\pi(t)\left[\mu \mathrm{d} t+\beta S^{\gamma}(t) \mathrm{d} Z^{(2)}(t)\right]+[W(t)-\pi(t)] k \mathrm{~d} t\right. \\
& \left.+\eta p(t) a \mathrm{~d} t+b p(t) \mathrm{d} Z^{(1)}\right\}+\frac{1}{2} \frac{\partial^{2} V}{w^{2}}\left\{\left[\pi^{2}(t)(t) \beta^{2} S^{2(\gamma+1)}(t)\right.\right. \\
& \left.\left.+2 \beta b \rho S^{2 \gamma}(t) \pi(t) p(t)+b^{2} p^{2}(t)\right] \mathrm{d} t\right\}
\end{aligned}
$$

for which we get the H-J-B equation

$$
\begin{aligned}
& V_{t}+\{\mu \pi(t)+[W(t)-\pi(t)] k+\eta p(t) a\} V_{w} \\
& +\frac{\pi^{2}(t)(t) \beta^{2} S^{2 \gamma}(t)+2 \beta b \rho S^{\gamma}(t) \pi(t)+b^{2} p^{2}(t)}{2} V_{w w}=0
\end{aligned}
$$

Applying (18)-(20) in (27), give the new H-J-B equation

$$
\begin{aligned}
& \frac{-1}{\phi} h^{\prime}+\{\{\mu \pi(t)+[W(t)-\pi(t)] k+\eta p(t) a\} \\
& \left.-\frac{\phi\left[\pi^{2}(t)(t) \beta^{2} S^{2 \gamma}(t)+2 \beta b \rho S^{\gamma}(t) \pi(t) p(t)+b^{2} p^{2}(t)\right]}{2}\right\} h=0
\end{aligned}
$$

The differentiation of (28) with respect to $\pi(t)$, gives 


$$
g(\mu-k)-\frac{\phi}{2}\left[2 \pi(t)(t) \beta^{2} S^{2 \gamma}(t)+2 \beta b \rho S^{\gamma}(t) p(t)\right]=0
$$

which when simplified gives the optimal value $\pi^{*}(t)$ as

$$
\pi^{*}(t)=\frac{(\mu-k)}{\phi \beta^{2} S^{2 \gamma}(t)}-\frac{b \rho p(t)}{\beta S^{\gamma}(t)}
$$

which is the reinsurer's optimal investment strategy for the risky asset. This is a function the risky asset's volatility and the reinsured proportion.

Also, when Equation (28) is differentiated with respect to $(t)$, we get

$$
\eta a-\phi\left[\beta b \rho S^{\gamma}(t) \pi(t)+b^{2} p(t)\right]=0
$$

from which we obtain the optimal reinsured proportion as

$$
p^{*}(t)=\frac{\eta a}{\phi b^{2}}-\frac{\rho \beta S^{\gamma}(t) \pi(t)}{b} .
$$

This is the optimal proportion the reinsurer accepts for reinsurance. It depends on the risky asset's volatility and the amount invested on the risky asset.

\subsection{The Effect of Correlation of the Brownian Motions}

This section expresses the impact of the correlation of Brownian motions as follows:

\subsubsection{The Case of the Investment Strategies}

We compare (23), when the Brownian motions do not correlate,

$$
\pi_{n c}^{*}(t)=\frac{(\mu-k)}{\phi \beta^{2} S^{2 \gamma}(t)}
$$

and (30) when the Brownian motions correlate,

$$
\pi_{c}^{*}(t)=\frac{(\mu-k)}{\phi \beta^{2} S^{2 \gamma}(t)}-\frac{b \rho p(t)}{\beta S^{\gamma}(t)},
$$

It can be observed that

$$
\pi_{c}^{*}(t)=\pi_{n c}^{*}(t)-\frac{b \rho p(t)}{\beta S^{\gamma}(t)}
$$

The investment strategy when Brownian motions do not correlate is greater than the strategy when the Brownian motions correlate by the fraction, $\frac{b \rho p(t)}{\beta S^{\gamma}(t)}$, where there is positive correlation.

If is $\rho$ negative, that is

$$
\rho=-\varepsilon
$$

we have

$$
\pi_{c}^{*}(t)=\pi_{n c}^{*}(t)+\frac{b \varepsilon p(t)}{\beta S^{\gamma}(t)}
$$

which implies that the optimal investment strategy when Brownian motions do 
not correlate is less than the optimal investment strategy when Brownian motions correlate with the amount $\frac{\varepsilon b p(t)}{\beta S^{\gamma}(t)}$.

Also, if

$$
\rho=1
$$

we have

$$
\pi_{c}^{*}(t)=\pi_{n c}^{*}(t)-\frac{b p(t)}{\beta S^{\gamma}(t)}
$$

Equation (37) shows that the optimal investment strategy when Brownian motions correlate is less than the optimal investment strategy when Brownian motions do not correlate by the amount $\frac{b p(t)}{\beta S^{\gamma}(t)}$. This situation is reversed when $\rho=-1$, giving

$$
\pi_{c}^{*}(t)=\pi_{n c}^{*}(t)+\frac{b p(t)}{\beta S^{\gamma}(t)}
$$

\subsubsection{The Case of the Reinsured Proportion}

Also comparing the optimal reinsured proportion as in (25), when the shocks do not correlate,

$$
p_{n c}^{*}(t)=\frac{a \eta}{\phi b^{2}}
$$

and (32) when the Brownian motions correlate,

$$
p_{c}^{*}(t)=\frac{a \eta}{\phi b^{2}}-\frac{\beta \rho S^{\gamma}(t) \pi(t)}{b},
$$

it can be seen that

$$
p_{n c}^{*}(t)=p_{c}^{*}(t)+\frac{\beta \rho S^{\gamma}(t) \pi(t)}{b}
$$

The optimal reinsured proportion when Brownian motions do not correlate is greater than the reinsured proportion when the Brownian motions correlate by $\frac{\beta \rho S^{\gamma}(t) \pi(t)}{b}$, which is a function of the price of the risky asset and the amount made available for investment in the risky asset. This is the case of $\rho$ being positive.

If $\rho$ is negative as in (34), we get

$$
p_{n c}^{*}(t)=p_{c}^{*}(t)-\frac{\beta \varepsilon S^{\gamma}(t) \pi(t)}{b}
$$

a change of the situation given in (39).

Applying (36) in (39), we get

$$
p_{n c}^{*}(t)=p_{c}^{*}(t)+\frac{\beta S^{\gamma}(t) \pi(t)}{b}
$$

and 


$$
p_{n c}^{*}(t)=p_{c}^{*}(t)-\frac{\beta S^{\gamma}(t) \pi(t)}{b}
$$

if $\rho=-1$.

\subsection{Findings}

1) The case of investment strategies: we found the investment strategies differ by the fraction, $\frac{b \rho p(t)}{\beta S^{\gamma}(t)}$, that is a function of the volatility of the risky asset and the reinsured proportion.

2) The case of the reinsured proportion: it found that the optimal reinsured proportion is a function of price of the risky asset and the amount made available for investment in the risky asset, given by $\frac{\beta \rho S^{\gamma}(t) \pi(t)}{b}$.

\section{Conclusions}

We considered the optimal investment problem for a reinsurer who grants insurance cover to insurance investors as proportional reinsurance purchase. It was assumed that claim process is driven by the constant elasticity of variance model. The reinsurer makes investments in two assets: a risk-free (bond) asset and a risky (stock) asset. The reinsurer aims at obtaining an optimal investment strategy and optimal proportion he can accept for reinsurance considering the correlation and none correlation of the Brownian motions, when he has exponential utility preference.

The application of the Ito's lemma gave room to obtaining the required Hamilton-Jacobi-Bellman Equations (HJB). The application of suitable conjecture based on the homogeneity of the objective function, the restriction and the terminal condition helped in our obtaining the required optimal investment strategy and optimal reinsured proportion.

We found that the optimal investment strategies differed by the fraction, $\frac{b \rho p(t)}{\beta S^{\gamma}(t)}$, that is a function of the volatility of the risky asset and the reinsured proportion for the case of correlation and none correlation of the Brownian motions. It was also found that the optimal reinsured proportion is a function of price of the risky asset and the amount made available for investment in the risky asset, given by $\frac{\beta \rho S^{\gamma}(t) \pi(t)}{b}$.

It is recommended that dependency on horizon, volatility of the risky asset, price of the risky asset and the reinsured proportion should be adequately monitored if the reinsurer should be solvent.

\section{Conflicts of Interest}

The author declares no conflicts of interest regarding the publication of this paper. 


\section{References}

[1] Browne, S. (1995) Optimal Investment Policies for a Firm with a Random Risk Process: Exponential Utility and Minimizing the Probability of Ruin. Mathematics of Operations Research, 20, 937-958. https://doi.org/10.1287/moor.20.4.937

[2] Liang, Z.B. and Bayraktar, E. (2014) Optimal Reinsurance and Investment with Un-observable Claim Size and Intensity. Insurance Mathematics and Economics, 55, 156-166. https://doi.org/10.1016/j.insmatheco.2014.01.011

[3] Shen, Y. and Wei, J.Q. (2016) Optimal Investment-Consumption-Insurance with Random Parameters. Scandinavian Actuarial Journal, 2016, 37-62. https://doi.org/10.1080/03461238.2014.900518

[4] Deng, Y., Zhou, J. and Huang, Y. (2015) Optimal Proportional Reinsurance and Investment for a Constant Elasticity of Variance Model under Variance Principle. $A c$ ta Mathematica Scientia, 35, 303-312. https://doi.org/10.1016/S0252-9602(15)60002-9

[5] Gao, J. (2009) Optimal Investment Strategy for Annuity Contracts under the Constant Elasticity of Variance (CEV) Model. Insurance: Mathematics and Economics, 45, 9-18. https://doi.org/10.1016/j.insmatheco.2009.02.006

[6] Ihedioha, S.A. and Osu, B.O. (2015) Optimal Probability of Survival of an Insurer and a Reinsurer under Proportional Reinsurance and Power Utility Preference. International Journal of Innovation in Science and Mathematics, 3, 2347-9051.

[7] Li, D., Rong, X. and Zhao, H. (2015) Time Consistent Reinsurance-Investment Strategy for a Mean-Variance Insurer under Stochastic Interest Rate Model and Inflation Risk. Insurance: Mathematics and Economics, 64, 28-44. https://doi.org/10.1016/j.insmatheco.2015.05.003

[8] Zhibin, L. and Guo, J. (2010) Optimal Proportional Reinsurance under Two Criteria: Maximizing the Expected Utility and Minimizing the Value at Risk. The ANZIAM Journal, 51, 449-463. https://doi.org/10.1017/S1446181110000878

[9] Gu, M., Yang, Y., Li, S. and Zhang, J. (2010) Constant Elasticity of Variance Model for Proportional Reinsurance and Investment Strategies. Insurance: Mathematics and Economics, 46, 580-587. https://doi.org/10.1016/j.insmatheco.2010.03.001 